\title{
Ecological Change Analysis of Lanzhou City Based on Remote Sensing Ecological Index
}

\author{
Yuhan Huang $^{\mathrm{a}, \mathrm{b}}$, Haowen Yan ${ }^{\mathrm{a}, \mathrm{b}, *}$ \\ ${ }^{a}$ Faculty of Geomatics, Lanzhou Jiaotong University, Lanzhou 730070, China, 435845463@qq.com, H. Yan, \\ haowen2010@gmail.com \\ ${ }^{b}$ Gansu Provincial Engineering Laboratory for National Geographic State Monitoring, Lanzhou 730070, China \\ 435845463@qq.com,H.Yan, haowen2010@gmail.com \\ * Corresponding author: H. Yan, haowen2010@gmail.com
}

Keywords: Ecological environment quality; RSEI; Principal component analysis; Lanzhou City

\begin{abstract}
Lanzhou City is the capital of Gansu Province and located in the semi-arid of northwest China. The Yellow River passing through the inner city from west to east, which has formed a special ecological environment. In recent years, the economic level of Lanzhou City has continued to develop, and the degree of urbanization has been continuously improved, which has had a certain impact on the ecological environment of the city. This paper used the Remote Sensing Ecology Index (RSEI) model (Xu, H.Q.,2013) to assess the ecological changes of the four major urban areas Chengguan District, Qilihe District, Anning District and Xigu District of Lanzhou from 2013 to 2017 , and evaluate the current ecological environment of the city to provide a basis for the sustainable development of the city.
\end{abstract}

During this study, Landsat8 OLI images were acquired on 2013-08-01and 2017-08-03 from Geospatial Data Cloud site, Computer Network Information Center, Chinese Academy of Sciences(http://www.gscloud.cn), over a period of 5 years. In order to ensure the accuracy of the results, the two-stage images were separately subjected to radiometric calibration and atmospheric correction. The two images were registered by the quadratic polynomial and the nearest neighbor pixel method, so that the root mean square error is controlled within 0.5 pixels. The RESI evaluation model was used to quickly evaluate the ecological status of Lanzhou City. The model uses principal component analysis to integrate four indicators, which are the normalized difference vegetation index (NDVI), normalized difference built-up and soil index (NDBSI), wetness component of the tasseled cap transformation (Wet), and land surface temperature (LST). Therefore, the model overcomes the shortcomings of single indicator evaluation. Because the study area is based on the city, the normalized difference built-up and soil index was replaced by the building index (IBI). The calculation formulas of each index are shown in Table 1.

\begin{tabular}{|l|c|}
\hline Index & computational formula \\
\hline NDVI & $\left(\rho_{N I R}-\rho_{R}\right) /\left(\rho_{N I R}+\rho_{R}\right)$ \\
\hline WET & $C_{1} \rho_{B}+C_{2} \rho_{G}+C_{3} \rho_{R}+C_{4} \rho_{N I R}+C_{5} \rho_{S W I R 1}+C_{6} \rho_{\text {SWIR } 2}$ \\
\hline LST & $\mathrm{LST}=\mathrm{T} /[1+(\lambda T / \rho) \ln \varepsilon]$ \\
& $\mathrm{T}=K_{2} / \ln \left(K_{1} / L_{10}+1\right)$ \\
\hline IBI & $\begin{array}{c}\left.\frac{2 \rho_{10}=\text { gain } \times D N+b i a s}{\rho_{S W I R 1}+\rho_{N I R}}-\rho_{N I R} /\left(\rho_{N I R}+\rho_{R}\right)-\frac{\rho_{G}}{\rho_{G}+\rho_{S W I R}}\right] /\left[\frac{2 \rho_{S W I R 1}}{\rho_{S W I R 1}+\rho_{N I R}}+\right. \\
\left.\frac{\rho_{N I R}+\rho_{R}}{\rho_{G}+\rho_{S W I R 1}}\right]\end{array}$ \\
\hline
\end{tabular}

Table 1 evaluation index calculation formula

In the table, where the $\rho_{\mathrm{i}}$ is the planetary reflectance of each band in the OLI sensors respectively. In the Landsat8 OLI image, $C_{1}=0.1511, C_{2}=0.1973, C_{3}=0.3283, C_{4}=0.3407, C_{5}=-0.7117, C_{6}=-0.4559$. In the calculation of land surface temperature, $\mathrm{L}_{10}$ is the radiation value of the band 10 of Landsat OLI; DN is the pixel gray value; gain and bias are the band gain value and offset value, gain $=3.342 \times 10-4$, bias $=0.1$; $\mathrm{T}$ is the temperature value of the sensor; $\mathrm{K}_{1}, \mathrm{~K}_{2}$ are calibration parameters, $\mathrm{K}_{1}=774.89 \mathrm{~W} /\left(\mathrm{m}^{2} . \mathrm{sr} . \mu \mathrm{m}\right), \mathrm{K}_{2}=1321.085 \mathrm{~K}$; LST is the surface temperature, $\lambda$ is the central wavelength of the thermal infrared band, $\rho=1.438 \times 10-2 \mathrm{~m} . \mathrm{K}, \varepsilon$ is the emissivity of the ground object, and its value is estimated by NDVI according to Sobrino's model. 


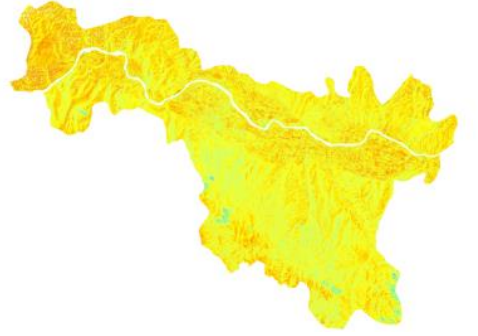

a.2013

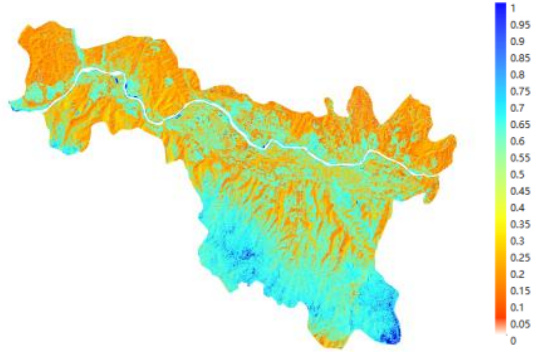

b.2017

Figure 1. Spatial distribution of RSEI in Lanzhou City.

Fig.1 revealed the spatial distribution of RSEI in Lanzhou City. The results of the experiments presented the average RSEI value increased from 0.14 in 2013 to 0.17 in 2017 . From the figure, we can see clearly that the overall ecological environment of Lanzhou City was poor in 2013. The area where the RSEI value is below 0.4 accounted for $86 \%$ of the total area; the average value of RSEI in 2017 has increased. But a notable characteristic of the distribution in RSEI was that the environmentally poor areas were gathered in the centre in 2017. Due to the development of urban construction in the inner city, the ecological environment has deteriorated and the index value has decreased.

RSEI is based on real-time remote sensing images and accordingly more capable of quick evaluation of temporal and spatial changes of ecological environment quality. It uses principal component analysis to integrate four indicators that have a significant impact on the ecological environment, avoiding the interference of human factors and quickly assessing changes in the urban ecological environment. The research on the ecological environment changes of Lanzhou shows that: (1) Overall, the ecological environment of Lanzhou has slightly improved from 2013 to 2017. (2) The ecological environment quality of inner city has declined may be attributed to the increasingly human activities. (3) The analysis results show that each of the selected indicators is key to indicating the ecological environment quality, and the model prediction result reveals that the control of barren and dry surfaces is a critical step for improving the ecological environment quality.

The work was financially supported by the National Key R\&D Program of China (2017YFB0504203), the National Natural Science Foundation of China (No. 41671447). 